\title{
A receptor like kinase gene with expressional responsiveness on Xanthomonas oryzae pv. oryzae is essential for Xa21-mediated disease resistance
}

\author{
Haitao Hu ${ }^{1,2+}$, Jing Wang ${ }^{1,2+}$, Chan Shi ${ }^{1,2+}$, Can Yuan ${ }^{1,2}$, Chunfang Peng ${ }^{1,2}$, Junjie Yin ${ }^{1,2}$, Weitao Li ${ }^{1,2}$, Min He ${ }^{1,2}$,
} Jichun Wang ${ }^{1,2}$, Bintian Ma ${ }^{1,2}$, Yuping Wang ${ }^{1,2}$, Shigui Li $i^{1,2,3}$ and Xuewei Chen ${ }^{1,2,3^{*}}$

\begin{abstract}
Background: Leucine-rich repeat receptor-like kinases (LRR-RLKs) represent a large class of proteins in regulating plant development and immunity. The LRR-RLK XA21 confers resistance to the bacterial disease caused by the pathogen of Xanthomonas oryzae pv. oryzae (Xoo). Several XA21 binding proteins have been characterized, however the early events governing XA21 signaling have not been fully elucidated.

Results: Here we report the identification of one LRR-RLK gene (XIK1) whose expression is induced rapidly upon the infection with the pathogen of Xoo. Expression pattern analysis reveals that XIK1 is preferentially expressed in reproductive leaves and panicles, and that expression is associated with plant development. By using RNA interference (RNAi), we silenced the expression of XIK1 in rice with Xa21 and found that reduced expression of XIK1 compromised disease resistance mediated by XA21. In addition, we found that the expression of the downstream marker genes of pathogen associated molecular pattern (PAMP) triggered immunity (PTI) in rice was compromised in Xa21 plants silenced for XIK1.
\end{abstract}

Conclusion: Our study reveals that the LRR-RLK gene XIK1 is Xoo-responsive and positively regulates Xa21-mediated disease resistance.

Keywords: LRR-RLK; Xa21; Disease resistance; Xanthomonas oryzae pv.oryzae

\section{Background}

Receptor-Like Kinases (RLKs) represent one of the largest protein families in plants, with more than 600 members in Arabidopsis (Shiu and Bleecker 2001) and 1000 members in rice (Shiu et al. 2004). A typical RLK protein contains extracellular structure, and transmembrane and kinase domains (Greeff et al. 2012). Based on the variation of the N-terminal domains, RLKs are divided into more than 44 subclasses (Gish and Clark 2011). The leucine-rich repeat (LRR)-RLK subclass is the largest, with 165 members in Arabidopsis (Shiu and Bleecker 2001) and 292 members in rice (Shiu et al. 2004). The LRR domain is a 24 residues-

\footnotetext{
* Correspondence: xwchen88@163.com

${ }^{\dagger}$ Equal contributors

${ }^{1}$ Rice Research Institute, Sichuan Agricultural University at Wenjiang,

Chengdu, Sichuan 611130, China

${ }^{2}$ State Key Laboratory of Hybrid Rice, Sichuan Agricultural University at Wenjiang, Chengdu 611130, China

Full list of author information is available at the end of the article
}

containing motif rich for leucine or other hydrophobic amino acids. Each LRR-RLK contains one or more LRRs to form a pocket used for binding to various ligands (Kolade et al. 2006; Gish and Clark 2011).

Previous studies have revealed that several LRR-RLKs are involved in plant development and immunity. For examples, the Arabidopsis BRI1, CLAVATA1, ERECTA1 (Clark et al. 1993; Torii et al. 1996; Clark et al. 1997; Li and Chory 1997; Jinn et al. 2000), rice BRI1 (OsBRI1) (Li and Chory 1997), and Petunia inflate PRK1 (Mu et al. 1994), regulate plant growth and development. The Arabidopsis flagellin receptor FLS2, the EF-Tu receptor EFR (GomezGomez and Boller 2000; Zipfel et al. 2006), and the rice protein XA21 (Song et al. 1995) mediate immunity during plant-microbe interaction.

Xa21 encodes a protein of rice LRR-RLK XII subclass and confers resistance against diverse strains of Xoo (Song et al. 1995). Although the expression of $\mathrm{Xa21}$ is 
quite stable during rice development, XA21-mediated disease resistance is development-dependent (Mazzola et al. 1994). Previous studies have shown that several XA21 binding proteins (XBs) regulate XA21-mediated immunity. These XBs belong to different protein families: such as RING finger ubiquitin ligase (XB3), transcriptional factor OsWRKY62 (XB10), protein phosphatase 2C (XB15), ATPase (XB24), endoplasmic reticulum chaperone (BiP3) and PANK protein (XB25) (Chen et al. 2010; Peng et al. 2008; Park et al. 2010; Wang et al. 2006; Park et al. 2008; Jiang et al. 2013). Recently, we also reported that OsSERK2, the rice ortholog of BAK1, regulates XA21-mediated immunity in a mechanism distinct from that of BAK1 in regulation of FLS2- and EFR-mediated immunity in Arabidposis (Chen et al. 2014b). Even with these advances, the mechanism of XA21-mediated immunity is still largely unknown.

Here, we characterized XIK1 (LOC_Os02g34790), which encodes a LRR-RLK protein, through analysis of the Rice Oligonucleotide array database (ROAD: http://www. ricearray.org/). We found that the expression of XIK1 was slightly induced upon the inoculation with Xoo in Kitaake but more strongly in $\mathrm{Xa21}$ plants. We found that XIK1 is ubiquitously expressed in different rice tissues and preferentially in leaves and panicles. Transgenic Xa21 plants silenced for $X I K 1$ exhibit compromised disease resistance to $X o o$. We also found that the expression of XIK1 is increasing during rice development, which might be the reason why XA21-mediated disease resistance is developmentally dependent. In addition, we found that the expression of marker genes for pathogen associated molecular pattern (PAMP) triggered immunity (PTI) is reduced in Xa21 plants silenced for XIK1. Taken together, our study reveals that the expression of XIK1 is Xoo-responsive and that XIK1 positively regulates XA21-mediated immunity.

\section{Results}

Identification of XIK1

LRR-RLK is a large subgroup of the rice RLK family, of which, XA21, XA3/XA26 and OsSERK2 have been reported to regulate plant immunity against $\mathrm{Xoo}$, the causal agent of bacterial leaf blight. To determine if any other LRR-RLK is involved in the immune response against Xoo, we analyzed global transcriptional analysis on the expression pattern of rice LRR-RLK genes to search those with Xoo-responsiveness using the data available from the Rice Oligonucleotide array database (http://www.ricearray.org/). We identified eight LRR-RLK genes whose transcriptional expressions were rapidly (at $2 \mathrm{~h}$ post inoculation) up-regulated upon Xoo inoculation (Additional file 1: Figure S1). We then selected one gene, LOC_Os02g34790, for further study. The protein encoded by LOC_OsO2g34790 contains a typical signal peptide at the N-terminal, 18 repeated LRR motif and a kinase domain at the C-terminal (Figure 1). The phylogenetic tree analysis reveals that this

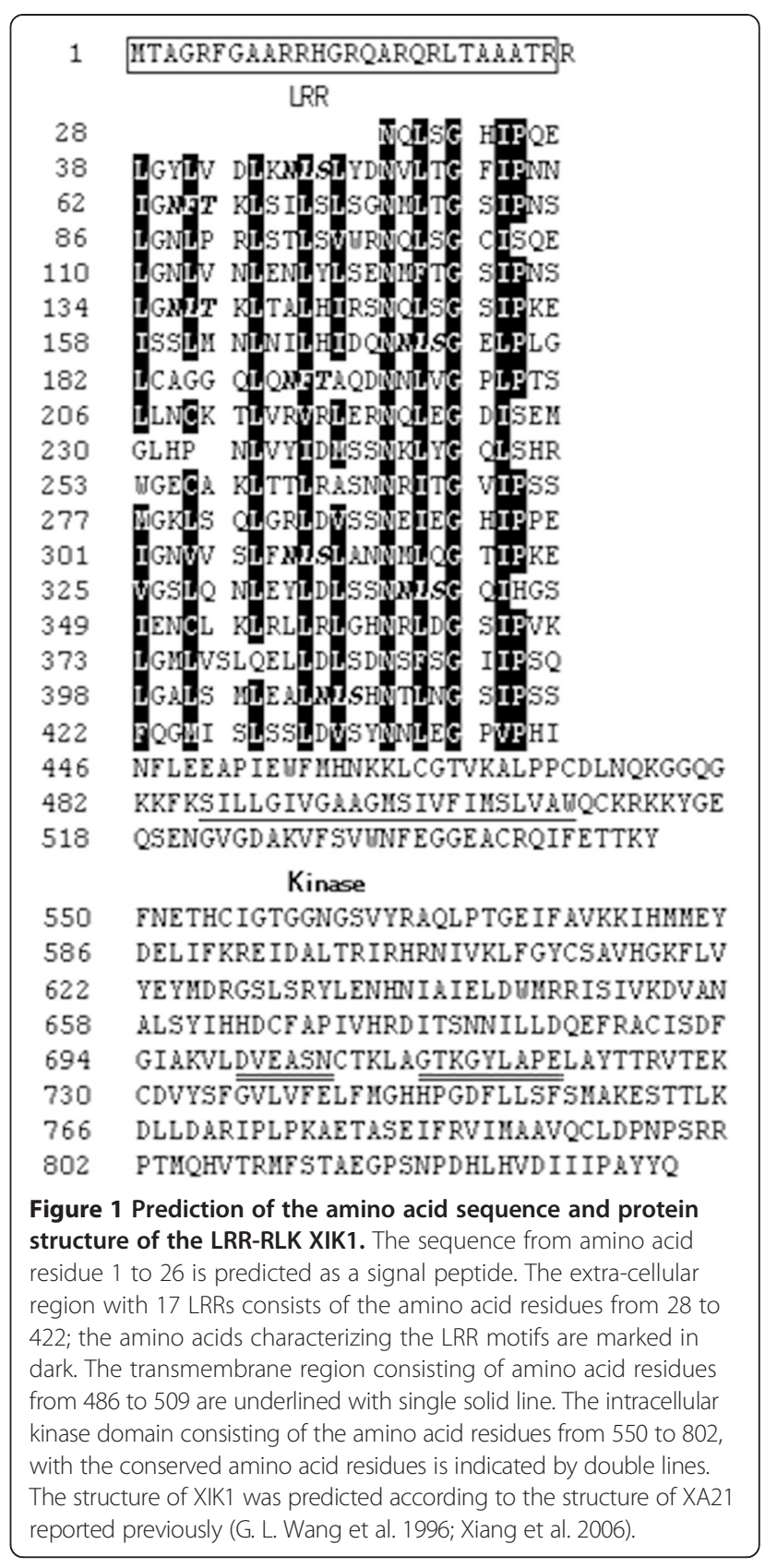

protein belongs to LRR II subfamily (Hwang et al. 2011). We then named this gene as Xoo-induced kinase 1 (XIK1).

\section{The expression pattern of XIK1}

We performed quantitative RT-PCR to measure the transcription levels of XIK1 in the rice tissues, including root, stem, leaf and panicle at the productive stage. We found that XIK1 was ubiquitously expressed in all of the tissues with a preference in leaves and panicles (Figure 2A). We then measured the expression of XIK1 in leaves collected from different developmental stages of rice and found that the expression of XIK1 increases during the development 

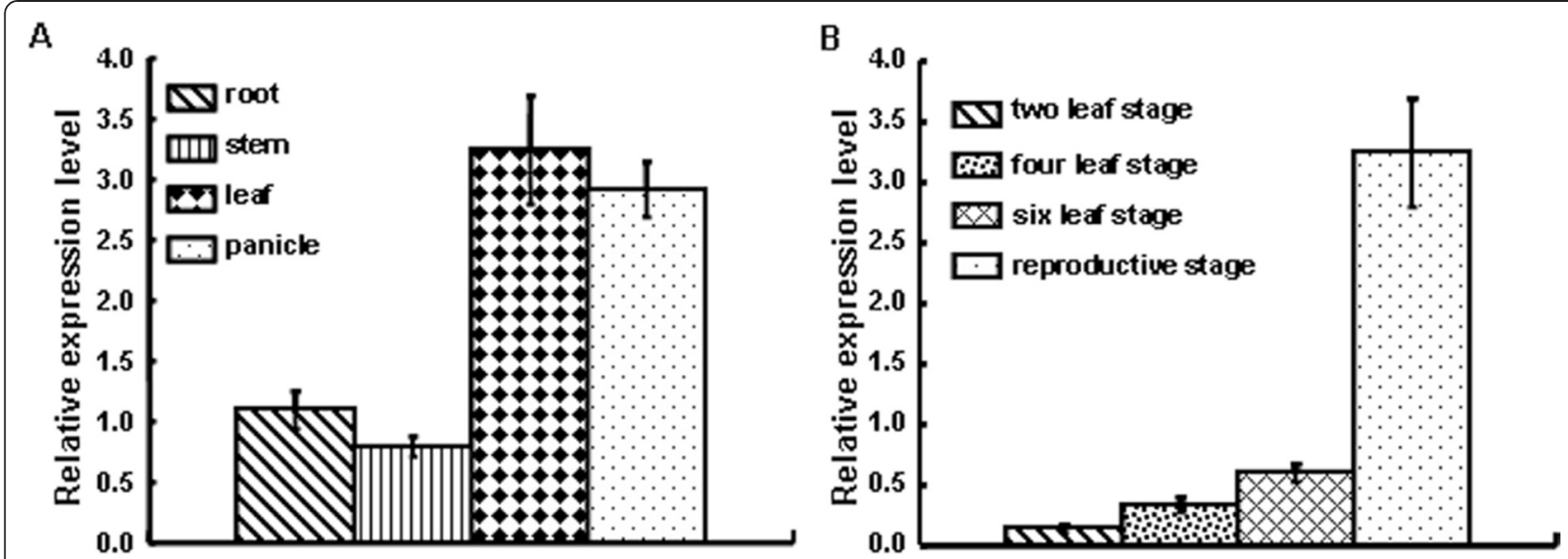

Figure 2 The expressions pattern of XIK1. (A). qRT-PCR was performed on CDNA synthesized from RNA samples extracted from rice cultivar Kitaake reproductive stage tissues. Expression of XIK1 was normalized to the expression of the ubiquitin 5 reference gene. Error bars indicating standard deviation (SD) obtained from three technical replicates. Three independent biological experiments were repeated with the similar results. (B). Determination of the transcriptional expression of XIK1 in leaves at different developmental stages as indicated. Gene expression of XIK1 was normalized to the expression of the ubiquitin5 reference gene. Error bars indicating SD obtained from three technical replicates. Three independent biological experiments were repeated with the similar results.

of rice (Figure 2B). These results suggest that XIK1 may function in both tissue- and development-dependent manners in rice.

The expression of XIK1 in Xa21 plants is induced by Xoo As the microarray data of XIK1 indicates that the expression of XIK1 in Nipponbare is responsive to Xoo, we tested whether it could also be induced in Xa21 plants (Peng et al. 2008). For this purpose, we collected the $X a 21$ rice leaves post the inoculation with $\mathrm{Xoo}$ or $\mathrm{H}_{2} \mathrm{O}$ (mock-treatment) and measured the expression of XIK1 using qRTPCR. We found that the expression of XIK1 was almost 1.5 folds of that obtained from mock treatment in Xa21 resistant lines (Figure 3 ). This result suggests that the expression of XIK1 in Xa21 plants is induced upon the inoculation with $X o o$.

\section{Silencing of XIK1 compromises the XA21-mediated resistance to Xoo}

Because the LRR-RLK XA21 functions as a pattern recognition receptor (PRR) to confer resistance to Xoo and XIK1 is rapidly induced by Xoo inoculation in Xa21 plants, we presumed that XIK1 regulates XA21-mediated immunity. To test this hypothesis, we introduced the XIK1Ri construct into ProA-Xa21 homozygous rice lines to silence the expression of XIK1 in Xa21 stable transgenic plants. ProA-Xa21 homozygous rice lines confer full resistance to the Xoo strain PXO99A (Chen et al. 2010). We obtained 12 independent double transgenic RNAi lines carrying both Xa21 and XIK1Ri and named them Xa21XIK1Ri (XXIK1Ri) lines. Three representative lines with reduced expression levels of XIK1 and stable expression level of the XIK1 homolog LOC_OsO2g34750 were found
(Additional file 2: Figure S2). Compared with that in the wild type ProA-Xa21 plants, the expression of XIK1 is reduced and is about $40 \%, 45 \%$ and $80 \%$ in the three XXIK1RNAi lines, XXIK1Ri-1, XXIK1Ri-3 and XXIK1Ri-8, respectively. Expression of LOC_OsO2g34750, with the highest identity (80.1\%) of cDNA sequence to LOC_Os02g34790, was not altered (Additional file 2: Figure S2). We inoculated the T0 generations of these transgenic lines, and observed that the $\mathrm{Xa21}$ plants

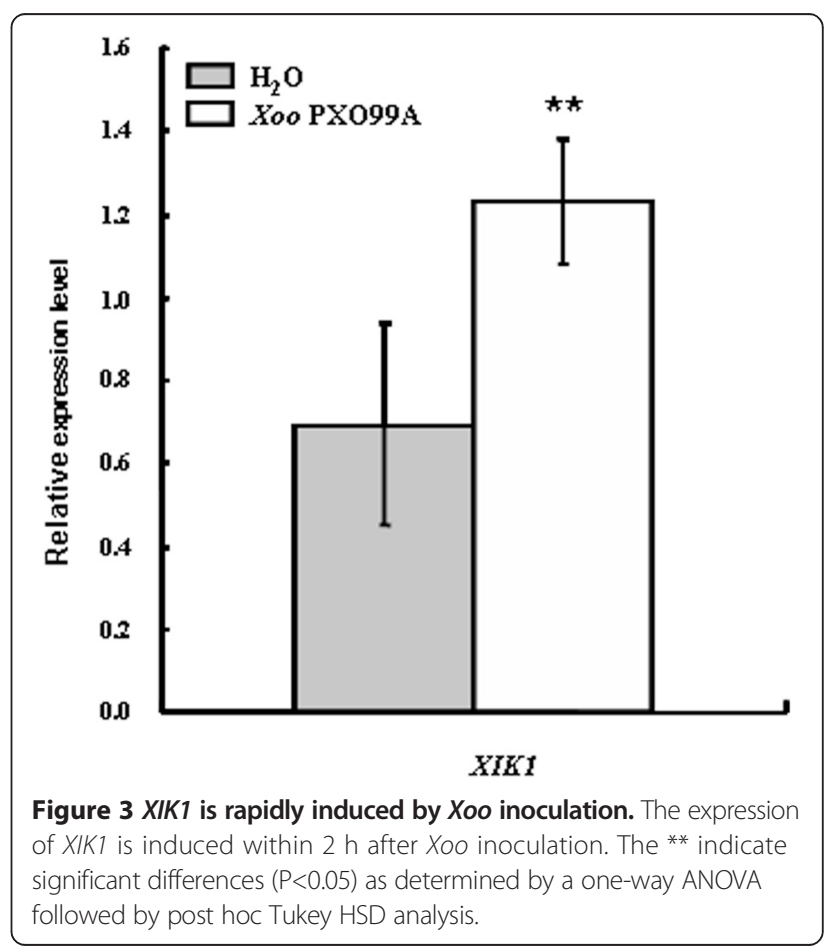


silenced for XIK1 exhibited compromised disease resistance to the Xoo strain PXO99A compared with the wild type ProA-Xa21 plants (Additional file 3: Figure S3A-C). We then analyzed the disease resistance of plants from $\mathrm{T} 1$ generations after inoculation with PXO99A. We found that the T1 plants segregated with partial resistance in some segregants and typical full resistance in others. The double transgenic plants carrying $\mathrm{Xa21}$ and silenced for XIK1 are partially resistant, showing typical long water-soaked lesions. The segregants carrying Xa21 but lacking of XIK1Ri confer full resistance to Xoo as do the wild type Xa21 plants (Additional file 3: Figure S3D). The Xa21 plants are highly resistant and the Kitaake plants are highly susceptible to the Xoo strain PXO99A as expected.

To characterize the effect of XIK1 on XA21-mediated disease resistance, we performed more detailed investigations using the plants (XXIK1Ri) homozygous for both $X a 21$ and $X X I K 1 R i$ derived respectively from three of the double transgenic lines. After we verified that the expression of XIK1 was reduced whereas the expression of Xa21 was not obviously changed in these RNAi lines (Additional file 4: Figure S4), we inoculated the plants with the Xoo strain PXO99AZ. At 14 days post inoculation (DPI), all of these three XXIK1Ri lines displayed longer lesions than the Xa21 control (Figure 4A-E). The average lesion length of the line XXIK1Ri-1 with the lowest expression level of XIK1 is about $4.29 \pm 0.69 \mathrm{~cm}$, with the longest lesion length among these three represent XIK1 silence lines. Meanwhile, XXIK1Ri-8 with the highest expression of XIK1 showed the shortest lesion length $(3.29 \pm 0.72) \mathrm{cm}$ among these three XIK1Ri lines. The positive control $\mathrm{Xa21}$ plants and the negative control Kitaake plants respectively show the average lesion length of $0.26 \pm 0.10 \mathrm{~cm}$ for the full resistance and $7.33 \pm 0.10 \mathrm{~cm}$ for the full susceptibility as expected (Figure 4F). These results suggest that these RNAi transgenic plants exhibit partial resistance, showing typical longer water-soaked lesions than ProA-Xa21 plants but shorter than Kitaake plants.

We then quantified bacteria accumulation in plant leaves after inoculation with $\mathrm{Xoo}$ at 0, 7, 14 and 21 DPI. We found that, at $21 \mathrm{DPI}$, the bacterial numbers in XXIK1Ri lines were almost 100 fold $\left(4.8 \times 10^{11}\right.$ for XXIK1Ri-1, $2.0 \times 10^{11}$ for XXIK1Ri-3 and $1.4 \times 10^{11}$ for XXIK1Ri-8) greater than in ProA-Xa21 control plants $\left(2.8 \times 10^{8}\right)$ (Figure 4G). These results are consistent with the results of disease lesion length data described above. The lesion lengths are longer and the bacterial numbers are greater when the expression level of XIK1 is lower in the XXIK1R $i$ lines (Additional file 2: Figure S2 and Additional file 4: Figure S4), suggesting that the resistance of the transgenic lines is in accordance with the expression level of XIK1. Taken together, these results suggest that reduced expression of XIK1 compromises XA21-mediated disease resistance and the LRR-LRK XIK1 plays essential roles in XA21-mediated full innate immunity in rice.

\section{Reduced expression of XIK1 compromises the downstream response of $\mathrm{PTI}$}

Previous reports have shown that the genes, OsKS4 (LOC_Os04g10060) and Os04g10010(LOC_Os04g10010), function as marker genes involved for the downstream responses associated PTI (Park et al. 2012; Chen et al. 2014a). Thus, we asked whether these two genes in Xa21 plants are also responsive to Xoo. For this purpose, we measured the expression of these two genes in rice plants post the inoculation with $\mathrm{Xoo}$ or $\mathrm{H}_{2} \mathrm{O}$ as a mock treatment. Compared with mock treatment, the expression levels of OsKS4 and Os04g10010 were induced more than two folds at 2 days post Xoo treatment (Figure 5). This result suggests that the XA21-mediated immune signaling was transduced downstream quickly. We then performed qRT-PCR analysis on the expression of the two genes using the leaf tissue samples collected from Kitaake, ProA-Xa21, and XXIK1Ri-1 plants without Xoo inoculation. We found that the expression levels of these two genes were respectively higher in ProA-Xa21 plants than that in Kitaake plants, suggesting that rice plants carrying Xa21 possess a higher level of basal PTI than Kitaake plants. More interestingly, we found that the expression of the two genes, OSKS4 and Os04g10010, were much reduced in XXIK1Ri-1 plants (3.6 \pm 0.4 fold and $2.8 \pm 0.2$ fold, respectivly) compared to ProA-Xa21 plants $(8.5 \pm 0.1$ fold and $4.0 \pm 0.7$ fold, respectivly) (Figure 6A). We then measured the expression of OSKS4 and Os04g10010 in ProA-Xa21 and XXIK1Ri-1 plants after Xoo inoculation. We found that the induction of both genes were inhibited in the plants silenced for XIK1 (Figure 6B and C). These results suggest that reduced expression of XIK1 compromises XA21-mediated immune response both before and after Xoo inoculation.

\section{Discussion}

Expression of the LRR-RLK gene XIK1 is responsive to Xoo inoculation

Although some resistant proteins have been reported to regulate rice resistance against Xoo, prior studies of RLK family proteins in rice were mainly focused on two RLKs: XA21 and XA3/XA26 (Chen and Ronald 2011). Phosphorylation events play critical roles for these RLKs to initiate the response triggered by PAMPs (Chen et al. 2010; Chen et al. 2014a). There are few reports showing that the expression of these RLKs is induced by pathogens and thus regulates the immune response (Century et al. 1999). Our study reveals that the RLK gene XIK1 was induced by Xoo and that the encoded protein XIK1 positively regulates XA21-mediated immunity (Figures 3 and 4 ). Previous 

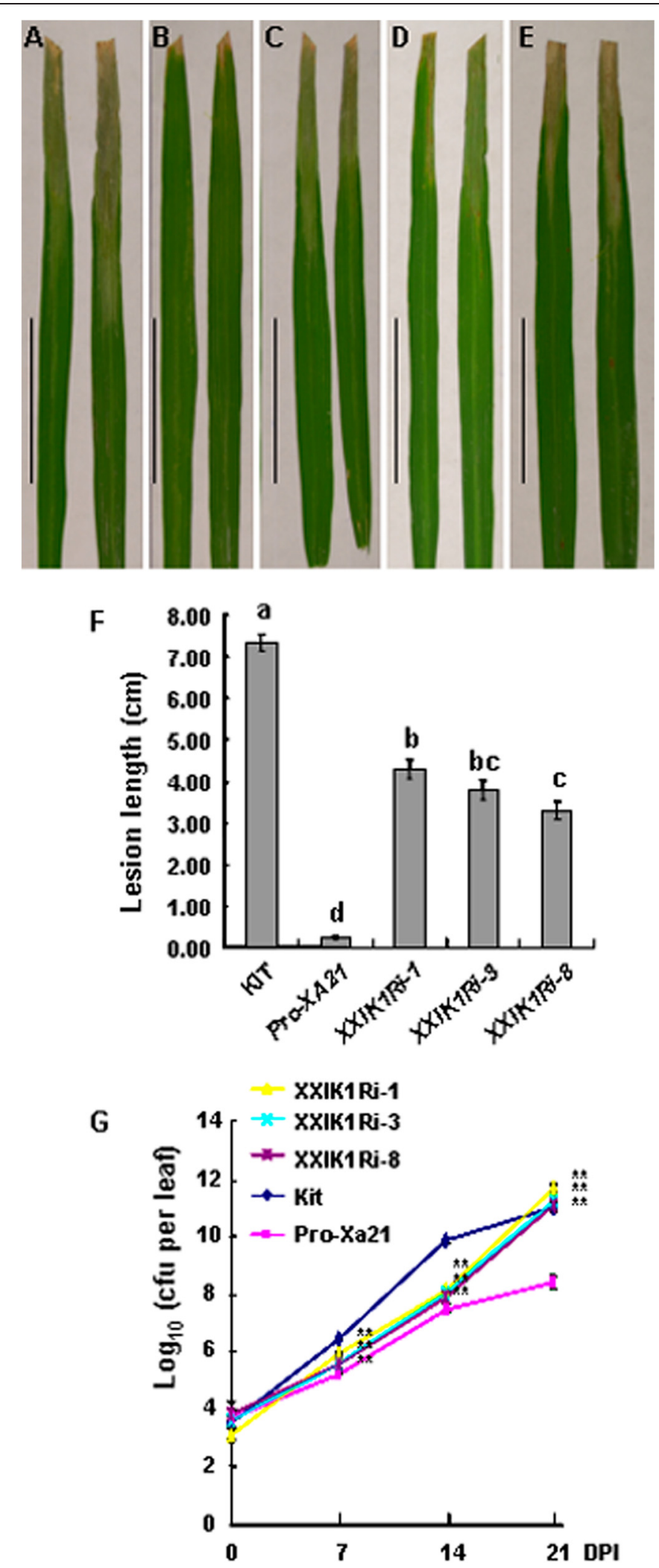

Figure 4 (See legend on next page.) 
(See figure on previous page.)

Figure 4 Determination of disease resistance of ProA-Xa21 plants silenced for XIK1. Six week-old plants were inoculated with the XoO strain PXO99A. The ProA-Xa21 and Kitaake (Kit) were used as resistant and susceptible controls, respectively. Photograph depicts the representative leaves from plants at 14 DPI. The leaves from Kitaake displayed the full susceptibility to Xoo (A) whereas the leaves from ProA-Xa21 displayed full resistance to Xoo (B). The panels $\mathbf{C}$, D, and $\mathbf{E}$ show the representative leaves from the three transgenic lines, XXIK1Ri-1, XXIK1Ri-3 and XXIK1Ri-8, respectively. All photographs were taken at $14 \mathrm{DPI}$. Bars $=5 \mathrm{~cm}$. (F), Lesion lengths of XXIK1Ri after inoculation with Xoo. Lesion length was measured at 14 DPI. Graph shows average lesion length \pm SD of at least 20 leaves from 10 independent plants homozygous for both Xa21 and XIKIRi. The letters indicate significant differences $(P<0.05)$ as determined by a one-way ANOVA followed by post hoc Tukey HSD analysis. (G), Bacterial cell numbers accumulated in ProA-Xa21 plants silenced for XIK1 after inoculation with Xoo. Bacterial cell numbers were counted at day $0,7,14,21$ post inoculations. Each data point represents the average \pm SD of six leaves from two independent plants. The ** indicate significant differences $(P<0.05)$ as determined by a one-way ANOVA followed by post hoc Tukey HSD analysis. These experiments were repeated at least three times with similar results.

studies have identified some proteins that play important roles in the regulation of XA21-mediated resistance. For examples, ubiquitin E3 ligase XB3 (Wang et al. 2006) and endoplasmic reticulum chaperone BiP3 were shown to keep the XA21 protein stable (Park et al. 2014). Protein phosphatase 2C XB15 (Park et al. 2008), ATPase $\mathrm{XB} 24$ and rice somatic embryogenesis receptor-like kinase 2 (OsSERK2) regulate the phosphorylation of XA21(Chen et al. 2010; Chen et al. 2014a), and the WRKY transcriptional factor XB10 regulates the XA21-mediated immune response through an unknown mechanism (Peng et al. 2008). However, the expressions of these genes were not obviously changed upon the inoculation of the Xoo strain PXO99A. Our finding that the expression of XIK1 was induced by Xoo suggests that some RLKs such as XIK1 regulate PTI via their transcriptional expression (Figure 3). This finding provides new insights in understanding the

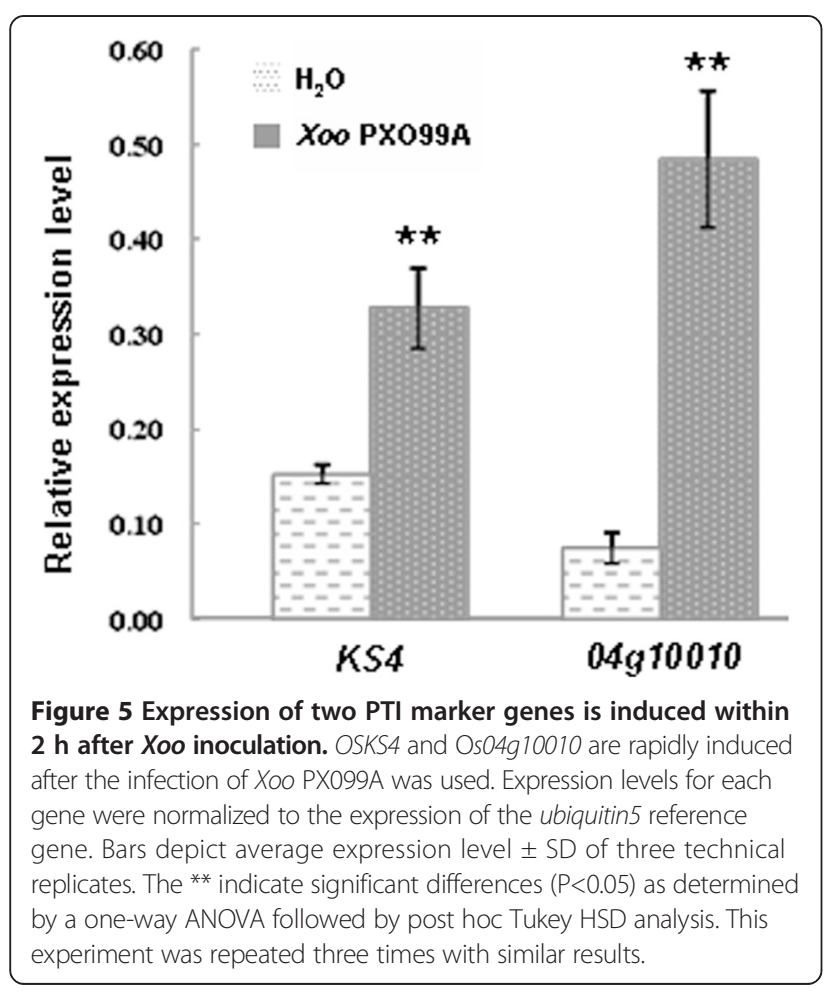

XA21-mediated PTI signaling and the RLK-mediated innate immunities. We also found that the PTI-related marker genes were down regulated in Xa21 plants silenced for XIK1 (Figures 5 and 6), suggesting that the expression of the genes involved in downstream of XA21-signaling are regulated by XIK1. Additionally, since expression of XIK1 is both tissue- and developmentally-dependent indicates that XIK1 regulates rice development and immunity tissue-specifically and developmentally (Figure 2). This result also provides the explanations why the disease resistance mediated by XA21 is developmentally-dependent.

We also performed a similar Xoo inoculation assay on four RNAi lines silenced for XIK1 in Kitaake rice and found that these RNAi lines were as susceptible as Kitaake plants (data not shown). Thus, our study suggests that XIK1 regulation of rice resistance to Xoo is XA21-dependent.

\section{XIK1 regulates early events in XA21-mediated immunity signaling}

LRR-RLKs consist of the largest RLK subfamily in plants with more than 165 members in Arabidopsis (Shiu and Bleecker 2001) and more than 292 members in rice (Shiu et al. 2004). In rice, the well studied LRR-RLK is XA21. Investigation of the molecular mechanisms of XA21-mediated immunity have shown that many proteins are required for XA21 to function (Peng et al. 2008; Park et al. 2010; Wang et al. 2006; Park et al. 2008; Jiang et al. 2013; Chen et al. 2014a). Our study reveals that the LRRRLK XIK1 positively regulates XA21-mediated immune signaling. As the expression of XIK1 is induced as early as within $2 \mathrm{~h}$ post $X o o$ inoculation and reduced expression of XIK1 compromises the expression of PTI-related genes, we hypothesize that XIK1 regulates the early events of XA21-mediated signaling. Because the XIK1 shares structural similarity with the LRR-RLK XA21, such as the extracellular LRR domain, a transmembrane domain that is required for the plasmembrane-localization of RLK proteins, and the intercellular active kinase domain, we suggest that XIK1 might work as a co-receptor of XA21 for PAMPs recognition and mediate the downstream signaling. As we could not detect the interaction between XIK1 and XA21 by using the intercellular domains of these two 

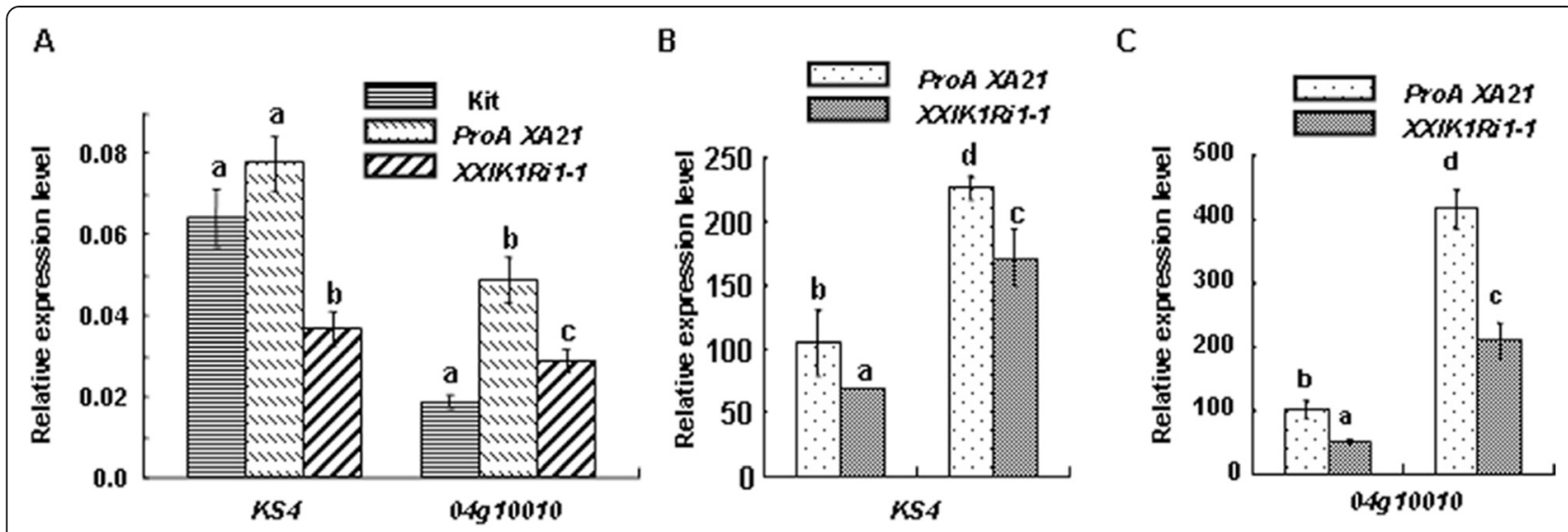

Figure 6 Silencing of XIK1 reduces the basal level of PTI marker genes in ProA-Xa21 plants. The relative expression level of two rice PTI marker genes, OSKS4 and Os04g10010, were determined by qRT-PCR using RNA extracted from rice leaves of six weeks old plants without Xoo inoculation $(\mathbf{A})$ and 2 days post $X_{0 o}$ inoculation $(\mathbf{B}, \mathbf{C})$. All data were normalized to the expression of the ubiqutin5 reference gene. The results of one representative experiment were shown. Error bars indicating SD of three technical replicates. Three independent biological experiments were repeated with the similar results. The letters indicate significant differences $(P<0.05)$ for each gene determined by a one-way ANOVA followed by post hoc Tukey HSD analysis.

RLKs in yeast (data not shown), the extracellular domains of XIK1 and XA21 or the binding of the PAMP might be required for their interaction. To understand the precise mechanism of XIK1 in regulation of XA21-mediated immunity, it is interesting to address these questions in our future work, including whether XIK1 forms a complex with XA21, whether the extra-cellular domains of these two RLKs, or pathogen inoculation are required for their interaction, and whether OsSERK2, a recently identified RLK essential for XA21-mediated immunity is required for XIK1 to function. We also suggest that XIK1 may regulate XA3/XA26, mediated immune response to Xoo, as XA3/XA26 belongs to LRR XII RLK with the similar structure of XA21.

In Arabidopsis, the LRR-LRKs, FLS2 and EFR also mediate PTI by recognizing the PAMPs, flagellin and elongation factor (EF-tu), respectively (Gomez-Gomez and Boller 2000; Zipfel et al. 2006). Previous studies have shown that FLS2- and EFR-mediated immunities share many similar processes as XA21, such as production of reactive oxygen species (ROS), activation of mitogen activated protein kinases (MAPKs), induction of pathogenesis-related genes (PRs) (Gomez-Gomez et al. 1999; Zhang and Zhou 2010; Zipfel et al. 2006) and the requirement of the SERK proteins (i.e. SERK3/BAK1 and SERK4/BKKI for FLS2 and EFR in Arabidopsis, and OsSERK2 for XA21 in rice) for their early signaling (Korner et al. 2013; Yang et al. 2011; Chen et al. 2014a). We hypothesize that the RLKs, encoded by At01g35710 and At04g08850, with high identities of amino acid sequence to XIK1, might also regulate the FLS2- and EFR-mediated early signaling through a similar mechanism as that of XIK1.

LysM-RLKs, another RLK subgroup, have been shown to mediate PTI in rice and Arabidopsis (Miya et al. 2007;
Shimizu et al. 2010; Chen and Ronald 2011). The LysM conserved motif of these receptors is reported to mediate plant-pathogen interactions by recognizing chitin molecules derived from fungal pathogens such as Magnaporthe oryzae (Chen and Ronald 2011). Thus, it would also be of great interest to determine whether XIK1 or other proteins with a similar structure of XIK1 regulate LysM-RLK-mediated immunity in the future work.

\section{Conclusion}

Our study revealed that the LRR RLK gene XIK1 is pathogen responsive and its expression is induced rapidly upon the inoculation with Xoo. Silencing of XIK1 comprises XA21-mediated resistance to Xoo and XIK1 positively regulates XA21-mediated resistance.

\section{Methods}

\section{Plant growth, Xoo inoculation and disease resistance} determination

All of the plants were grown in rice fields (Wenjiang, Chengdu, Sichuan, China). Rice Kitaake is used for transcriptional expression pattern analysis. The silencing construct of XIK1 (XIK1RNAi) was introduced into the Xa21 (ProA-Xa21) plants with full resistance to the Xoo strain PXO99A (Hopkins et al. 1992) to obtaine double transgenic plants containing Xa21 and XIK1RNAi. Plants were inoculated with the Xoo strain PXO99A. The inoculation and disease lesion length determination was performed according to the methods described previously (Song et al. 1995). Statistical analysis was performed using a one-way ANOVA followed by post hoc Tukey HSD. The leaves inoculated with Xoo were collected and prepared for transcriptional expression analyses as described below. 


\section{RNA extraction and real time RT-PCR analyses}

Total RNA was isolated from rice plant tissues using Invitrogen RNA isolation reaction, TRIzol (Invitrogen), following the procedures of the manual. The first strand cDNA was synthesized using the Takara reverse transcription kit (Takara). Quantitative real time PCR (qRT-PCR) was performed on a Bio-Rad CFX96 Real-Time System coupled to a C1000 Thermal Cycler (Bio-Rad). For qRTPCR reactions, the SsoFastEva Green Super mix was used. The gene expression levels relatively to the ubiquitin5 gene (LOC-06g46770) were analyzed using delta-delta ct method. All of the qRT-PCR primers are shown in Additional file 5: Table S1.

\section{Constructions}

For XIK1 RNAi construct, a 361 bp unique cDNA sequence (from $72 \mathrm{nt}$ to $432 \mathrm{nt}$ ) of XIK1Ri amplified by primer pair XIK1Ri-1/-2 (shown in Additional file 5: Table S1) and was cloned into_pCR ${ }^{\circ} 8^{\mathrm{m}} / \mathrm{GW} / \mathrm{TOPO}^{\circ}$ (Invitrogen) vector to create the construct $p$ CR8-XIK1Ri. $p$ CR8-XIK1Ri was then inserted into pANDA (Miki and Shimamoto 2004) through LR recombination to create the construct pANDA-XIK1Ri.

\section{Generation of transgenic rice plants}

The RNAi construct for XIK1, pANDA-XIK1Ri, was introduced into Xa21 plants through Agrobacterium-mediated transformation according to the method described previously (Chern et al. 2005). Because the ProA-Xa21 transgenic plants are mannose resistant, XIK1Ri transgenic plants in Xa21 genetic background were selected with hygromycin in our study.

\section{Accession numbers \\ RGAP: LOC_Os02g34790, LOC_Os02g34750}

\section{Additional files}

Additional file 1: Figure S1. Identification LRR-RLKs induced by $X_{0 O}$ inoculation in 2 hours. These genes were identified from the array data (http://www.ricearray.org/) of experiment "Comparative transcriptional profiling of rice undergoing infection by X.oryzae pv oryzae or by X.oryzae pv.oryzicola". We selected the LRR-RLKs which were specially induced by Xoo strain PXO99A in susceptible variety Nipponbare.

Additional file 2: Figure S2. Identification of transgenic plants with reduced expression of XIK1. (A) A special fragment was chosen for XIK1 RNAi construct. (B) The relative expression level of XIK1 was determined by qRT-PCR using RNA extracted from rice leaves of RNAi transgenic lines as indicated and ProA-Xa21 plants. All data were normalized to the expression of the ubiqutin 5 reference gene. The average expression level from one representative biological experiment was shown. Error bars indicate SD of three technical replicates. Three independent biological experiments were repeated with the similar results obtained.

Additional file 3: Figure S3. Determination of the resistance of the T0 and T1 generation of XIK1Ri transgenic plants to Xoo. Six weeks-old plants were inoculated with the Xoo strain PXO99A. The ProA-Xa21 and Kitaake (Kit) were used as resistant and susceptible controls, respectively. From
(A) through (C), TO Transgenic plants carrying XIKIRi developed long water-soaking lesions. Photograph depicts the representative leaves from the plants at $14 \mathrm{DPI}$. (D), Lesion length was measured 14 days post inoculation with Xoo strain PXO99A. "Ri(+)" indicates T1 segregants carrying the transgene XIK1Ri whereas "Ri(-)" indicates T1 segregants lacking of XIKIRi. This experiment was repeated three times with similar results.

Additional file 4: Figure S4. Expression level of $X_{a 21}$ in the plants silenced for XIK1 by qRT-PCR. All data were normalized to the expression of the ubiqutin5 reference gene.

Additional file 5: Table S1. The primers used in this study.

\section{Abbreviations}

DPI: Days post inoculation; XIK1: Xoo-induced kinase 1.

\section{Competing interests}

The authors declare that they have no competing interests

\section{Authors' contributions}

JW and XC conceived this study. HH, CS, CP, WL, MH, CY, JY, JW, BM, YW performed the experiments. HH, CS, WL, JW, SL and XC analyzed data. JW and XC wrote the manuscript. All authors approved the manuscript.

\section{Acknowledgments}

The authors thanks for the grants from National Natural Science Foundation of China (NSFC 31171622; 31371705), "Hundred Talents Plan" foundation of Sichuan, Youth foundation of Sichuan (13QNJJ0076) in China and Sichuan Agricultural University "High Talents" start-up foundation to X. W. Chen, the grants Specialized Research Fund for doctoral program of Higher Education (20135103120004) to J. Wang and the grants Specialized Research Fund for doctoral program of Higher Education (20125103120011) to W. T. Li. We also thank Dr. Pamela C Ronald from University of California at Davis for helpful discussion.

\section{Author details}

${ }^{1}$ Rice Research Institute, Sichuan Agricultural University at Wenjiang, Chengdu, Sichuan 611130, China. ${ }^{2}$ State Key Laboratory of Hybrid Rice, Sichuan Agricultural University at Wenjiang, Chengdu 611130, China. ${ }^{3}$ Collaborative Innovation Center for Hybrid Rice in Yangtze River Basin at Sichuan, Chengdu 611130, China.

Received: 16 July 2014 Accepted: 6 December 2014 Published online: 17 January 2015

\section{References}

Century KS, Lagman RA, Adkisson M, Morlan J, Tobias R, Schwartz K Smith A, Love J, Ronald PC, Whalen MC (1999) Short communication: developmental control of Xa21-mediated disease resistance in rice. Plant J 20(2):231-236

Chen X, Ronald PC (2011) Innate immunity in rice. Trends Plant Sci 16(8):451-459, doi:10.1016/j.tplants.2011.04.003

Chen X, Chern M, Canlas PE, Ruan D, Jiang C, Ronald PC (2010) An ATPase promotes autophosphorylation of the pattern recognition receptor XA21 and inhibits XA21-mediated immunity. Proc Natl Acad Sci U S A 107(17):8029-8034, doi:10.1073/pnas.0912311107

Chen X, Zuo S, Schwessinger B, Chern M, Canlas PE, Ruan D, Zhou X, Wang J, Daudi A, Petzold CJ, Heazlewood JH, Ronald PC (2014a) An XA21-associated kinase (OsSERK2) regulates immunity mediated by the XA21 and XA3 immune receptors. Mol Plant, doi:10.1093/mp/ssu003

Chen X, Zuo S, Schwessinger B, Chern M, Canlas PE, Ruan D, Zhou X, Wang J, Daudi A, Petzold CJ, Heazlewood JH, Ronald PC (2014b) An XA21-associated kinase (OsSERK2) regulates immunity mediated by the XA21 and XA3 immune receptors. Mol Plant 7(5):874-892, doi:10.1093/mp/ssu003

Chern M, Canlsa PE, Fitzgerald H, Ronald PC (2005) NRR, a negative regulator of disease resistance in rice that interacts with Arabidopsis NPR1 and rice NH1. The Plant Journal 43(5):623-635

Clark SE, Running MP, Meyerowitz EM (1993) CLAVATA1, a regulator of meristem and flower development in Arabidopsis. Development 119(2):397-418

Clark SE, Williams RW, Meyerowitz EM (1997) The CLAVATA1 gene encodes a putative receptor kinase that controls shoot and floral meristem size in Arabidopsis. Cell 89(4):575-585 
Gish LA, Clark SE (2011) The RLK/Pelle family of kinases. Plant J 66(1):117-127, doi:10.1111/j.1365-313X.2011.04518.x

Gomez-Gomez L, Boller T (2000) FLS2: an LRR receptor-like kinase involved in the perception of the bacterial elicitor flagellin in Arabidopsis. Mol Cell 5(6):1003-1011

Gomez-Gomez L, Felix G, Boller T (1999) A single locus determines sensitivity to bacterial flagellin in Arabidopsis thaliana. Plant J 18(3):277-284

Greeff C, Roux M, Mundy J, Petersen M (2012) Receptor-like kinase complexes in plant innate immunity. Front Plant Sci 3:209, doi:10.3389/fpls.2012.00209

Hopkins CM, White FF, Choi SH, Guo A, Leach JE (1992) Identification of a family of avirulence genes from Xanthomonas oryzae pv. oryzae. Mol Plant Microbe Interact 5(6):451-459

Hwang SG, Kim DS, Jang CS (2011) Comparative analysis of evolutionary dynamics of genes encoding leucine-rich repeat receptor-like kinase between rice and Arabidopsis. Genetica 139(8):1023-1032, doi:10.1007/s10709-011-9604-y

Jiang Y, Chen X, Ding X, Wang Y, Chen Q, Song WY (2013) The XA21 binding protein XB25 is required for maintaining XA21-mediated disease resistance. Plant J 73(5):814-823, doi:10.1111/tpj.12076

Jinn TL, Stone JM, Walker JC (2000) HAESA, an Arabidopsis leucine-rich repeat receptor kinase, controls floral organ abscission. Genes Dev 14(1):108-117

Kolade OO, Bamford VA, Ancillo Anton G, Jones JD, Vera P, Hemmings AM (2006) In vitro characterization of the cysteine-rich capping domains in a plant leucine rich repeat protein. Biochim Biophys Acta 1764(6):1043-1053, doi:10.1016/j. bbapap.2006.03.014

Korner CJ, Klauser D, Niehl A, Dominguez-Ferreras A, Chinchilla D, Boller T, Heinlein M, Hann DR (2013) The immunity regulator BAK1 contributes to resistance against diverse RNA viruses. Mol Plant Microbe Interact 26(11):1271-1280, doi:10.1094/MPMI-06-13-0179-R

Li J, Chory J (1997) A putative leucine-rich repeat receptor kinase involved in brassinosteroid signal transduction. Cell 90(5):929-938

Mazzola M, Leach JE, Nelson R, White FF (1994) Analysis of the interaction between Xanthomonas oryzae pv. oryzae and the rice cultivars IR24 and IR-BB21. Phytopathology, 84(8), 392-397. doi:10.1094/Phyto-84-392

Miki D, Shimamoto K (2004) Simple RNAi vectors for stable and transient suppression of gene function in rice. Plant Cell Physiol 45(4):490-495

Miya A, Albert P, Shinya T, Desaki Y, Ichimura K, Shirasu K, Narusaka Y, Kawakami N, Kaku H, Shibuya N (2007) CERK1, a LysM receptor kinase, is essential for chitin elicitor signaling in Arabidopsis. Proc Natl Acad Sci U S A 104 (49):19613-19618, doi:10.1073/pnas.0705147104

Mu JH, Lee HS, Kao TH (1994) Characterization of a pollen-expressed receptor-like kinase gene of Petunia inflata and the activity of its encoded kinase. Plant Cell 6(5):709-721, doi:10.1105/tpc.6.5.709

Park CJ, Peng Y, Chen X, Dardick C, Ruan D, Bart R, Canlas P, Ronald PC (2008) Rice $X B 15$, a protein phosphatase 2 , negatively regulates cell death and XA21-mediated innate immunity. PLoS Biol 6(9):e231, doi:10.1371/journal. pbio.0060231

Park CJ, Bart R, Chern M, Canlas PE, Bai W, Ronald PC (2010) Overexpression of the endoplasmic reticulum chaperone BiP3 regulates XA21-mediated innate immunity in rice. PLoS One 5(2):e9262, doi:10.1371/journal.pone.0009262

Park CJ, Chen S, Shirsekar G, Zhou B, Khang CH, Songkumarn P, Afzal AJ, Ning Y, Wang R, Bellizzi M, Valent B, Wang GL (2012) The Magnaporthe oryzae effector AvrPiz-t targets the RING E3 ubiquitin ligase APIP6 to suppress pathogen-associated molecular pattern-triggered immunity in rice. Plant Cell 24 (11): 4748-4762, doi:10.1105/tpc.112.105429

Park CJ, Song MY, Kim CY, Jeon JS, Ronald PC, Park CJ, Song MY, Kim CY, Jeon JS, Ronald PC (2014) Rice BiP3 regulates immunity mediated by the PRRs XA3 and XA21 but not immunity mediated by the NB-LRR protein, Pi5. Biochem Biophys Res Commun 448(1):70-75, doi:10.1016/j.bbrc.2014.04.093

Peng Y, Bartley LE, Chen X, Dardick C, Chern M, Ruan R, Canlas PE, Ronald PC (2008) OsWRKY62 is a negative regulator of basal and Xa21-mediated defense against Xanthomonas oryzae pv. oryzae in rice. Mol Plant 1(3):446-458, doi:10.1093/mp/ssn024

Shimizu T, Nakano T, Takamizawa D, Desaki Y, Ishii-Minami N, Nishizawa Y, Minami E, Okada K, Yamane H, Kaku H, Shibuya N (2010) Two LysM receptor molecules, CEBiP and OsCERK1, cooperatively regulate chitin elicitor signaling in rice. Plant J 64(2):204-214, doi:10.1111/j.1365-313X.2010.04324.X

Shiu SH, Bleecker AB (2001) Plant receptor-like kinase gene family: diversity, function, and signaling. Sci STKE (113):re22, doi:10.1126/stke.2001.113.re22

Shiu SH, Karlowski WM, Pan R, Tzeng YH, Mayer KF, Li WH (2004) Comparative analysis of the receptor-like kinase family in Arabidopsis and rice. Plant Cell 16 (5):1220-1234, doi:10.1105/tpc.020834
Song WY, Wang GL, Chen LL, Kim HS, Pi LY, Holsten T, Gardner J, Wang B, Zhai W, Zhu L, Fauquet C, Ronald PC (1995) A receptor kinase-like protein encoded by the rice disease resistance gene, Xa21. Science 270(5243):1804-1806

Torii KU, Mitsukawa N, Oosumi T, Matsuura Y, Yokoyama R, Whittier RF et al (1996) The Arabidopsis ERECTA gene encodes a putative receptor protein kinase with extracellular leucine-rich repeats. Plant Cell 8(4):735-746, doi:10.1105/tpc.8.4.735

Wang GL, Song WY, Ruan DL, Sideris S, Ronald PC (1996) The cloned gene, Xa21, confers resistance to multiple Xanthomonas oryzae pv. oryzae isolates in transgenic plants. Mol Plant Microbe Interact 9(9):850-855

Wang YS, Pi LY, Chen X, Chakrabarty PK, Jiang J, De Leon AL et al (2006) Rice XA21 binding protein 3 is a ubiquitin ligase required for full Xa21-mediated disease resistance. Plant Cell 18(12):3635-3646, doi:tpc.106.046730

Xiang Y, Cao Y, X U C, Li X, Wang S (2006) Xa3, conferring resistance for rice bacterial blight and encoding a receptor kinase-like protein, is the same as Xa26. Theor Appl Genet 113(7):1347-1355, doi:10.1007/s00122-006-0388-x

Yang DH, Hettenhausen C, Baldwin IT, Wu J (2011) The multifaceted function of BAK1/SERK3: plant immunity to pathogens and responses to insect herbivores. Plant Signal Behav 6(9):1322-1324

Zhang J, Zhou JM (2010) Plant immunity triggered by microbial molecular signatures. Mol Plant 3(5):783-793, doi:10.1093/mp/ssq035

Zipfel C, Kunze G, Chinchilla D, Caniard A, Jones JD, Boller T et al (2006) Perception of the bacterial PAMP EF-Tu by the receptor EFR restricts Agrobacteriummediated transformation. Cell 125(4):749-760, doi:10.1016/j.cell.2006.03.037

\section{Submit your manuscript to a SpringerOpen ${ }^{\circ}$ journal and benefit from:}

- Convenient online submission

- Rigorous peer review

- Immediate publication on acceptance

- Open access: articles freely available online

- High visibility within the field

- Retaining the copyright to your article

Submit your next manuscript at $>$ springeropen.com 